\title{
基于激光红外检测信号的表面裂纹定量重构
}

杨阳，邱金星，刘皓晨，裴翠祥，陈振茂

(陕西省无损检测与结构完整性评价工程技术研究中心, 机械结构强度与振动国家重点实验室,

西安交通大学, 陕西 西安 710049)

\begin{abstract}
摘要: 激光红外检测方法作为一种新型的远距离无损检测技术, 对于表面裂纹的检测具有很高的效 率。本文基于频域叠加法和数据库策略实现了激光激励下表面温度的快速数值模拟, 研究了裂纹尺 寸与温度分布间的关系，在时域与空域验证了快速数值方法的有效性。为基于红外检测信号实现裂 纹尺寸的定量重构，选取合适的特征量并且建立了基于确定论方法的裂纹重构算法。最后，搭建了 红外检测实验系统, 进行了平板试件裂纹红外检测实验, 基于实验信号实现了裂纹的定量重构, 验 证了重构算法的有效性。
\end{abstract}

关键词: 激光红外检测; 表面裂纹; 定量重构; 确定论方法

中图分类号: TP274.52; TP391.41 文献标识码: A 文章编号 : 1001-8891(2020)02-0152-06

\section{Reconstruction of Surface Cracks with Laser Spot Thermography Signals}

YANG Yang, QIU Jinxing, LIU Haochen, PEI Cuixiang, CHEN Zhenmao

(Shaanxi Engineering Research Center of NDT and Structural Integrity Evaluation,

State Key Laboratory for Strength and Vibration of Mechanical Structures, Xi'an Jiaotong University, Xi'an 710049, China)

\begin{abstract}
Laser spot thermography(LST) is a novel remote nondestructive testing technology. It can efficiently detect surface cracks. In this paper, a fast simulation method based on frequency domain summation and database strategy is developed to simulate the heat flow generated by the laser spot source and to investigate the relationship between crack size and temperature distribution. First, the feasibility of the new method is validated through comparison with a conventional method for both the time and the spatial domains. Second, suitable characteristic parameters are introduced, and a reconstruction algorithm based on a deterministic inversion method is developed. Finally, an infrared thermography testing system is established, and LST signals of surface cracks in a plate specimen are measured. The feasibility of the inversion method is validated by the accurate reconstruction result of crack size.
\end{abstract}

Key words: laser spot thermography, surface crack, quantitative reconstruction, deterministic method

\section{0 引言}

为了确保关键设施结构安全运行, 对表面裂纹进 行检测十分重要, 需要在工程实际应用中采用无损检 测技术。目前应用较多的常规无损检测方法, 例如超 声检测、渗透检测和浴流检测, 存在不能远距离对结 构进行检测, 检测效率低下的问题 ${ }^{[1]}$ 。

作为一种新型的红外检测方法, 激光红外检测技 术运用激光热源对被测试件表面加热并利用红外相 机检测记录表面的温度分布图像 ${ }^{[2]}$ 。当激光光斑靠近
表面裂纹时, 其表面热流会因裂纹的存在而产生扰 动, 因此通过对红外图像的分析即可实现对表面裂纹 的检测。与常规的无损检测方法相比, 激光红外检测 技术具有远距离、非接触和高检测效率的优点 ${ }^{[3]}$ 。由 于采用激光点光源作为热源, 其热流主要沿表面传 播, 因此非常适合用于表面裂纹的检测 ${ }^{[4]}$ 。此外, 基 于表面温度信号, 该方法也有望实现表面裂纹的定量 重构。

本文根据激光红外检测的基本原理，并基于频域 叠加法和数据库策略, 实现了表面温度的快速数值模

收稿日期：2019-07-10；修订日期: 2019-12-11.

作者简介: 杨阳 (1997-), 男, 河南省三门峡市人, 西安交通大学航天航空学院硕士研究生, 主要从事红外无损检测理论和实验研究。E-mail: yang19970106@stu.xjtu.edu.cn。

通信作者: 陈振茂 (1964-), 男, 山西省怀仁市人, 西安交通大学航天航空学院教授, 主要从事无损检测和电磁固体力学研究。E-mail: chenzm@xjtu.edu.cn。 基金项目: 国家自然科学基金（51577139）。 
拟, 计算了不同裂纹尺寸下试件表面的温度分布。通 过引入合适的信号特征量, 基于确定论裂纹重构算法, 实现了基于激光红外检测信号的表面裂纹定量重构。 最后进行了红外检测实验, 基于实验信号完成了对试 件表面裂纹的定量重构, 验证了该方法的有效性。

\section{1 数值模拟方法}

\section{1 红外检测数值模拟理论}

激光红外检测的导热为瞬态传热过程。根据傅里 叶传热定律, 对于均匀、各向同性材料, 忽略其表面 辐射和对流, 并设内热源为零, 这时热传导方程可写 为如下形式:

$$
\rho c \dot{T}-\kappa \nabla^{2} T=Q
$$

式中: $\rho 、 c 、 \kappa$ 分别为材料的密度、比热容和热导率; $T$ 为温度; $Q$ 表示外部热激励。

温度场的边界条件可表示为:

$$
\left.\left(n_{x} \frac{\partial T}{\partial x}+n_{y} \frac{\partial T}{\partial y}+n_{z} \frac{\partial T}{\partial z}\right)\right|_{\Gamma}=-\frac{q}{\kappa}
$$

式中: $n_{x}, n_{y}, n_{z}$ 为边界法向量在 $x 、 y 、 z$ 方向上的分 量; $q$ 为激光热源的热流密度。根据有限元方法, 控 制方程可离散表达为如下形式:

$$
[\boldsymbol{K}]\{T\}+[\boldsymbol{C}]\left\{\frac{\partial T}{\partial t}\right\}=\{Q\}
$$

式中: $[K] 、[C]$ 分别为有限元模型导热系数矩阵和比 热系数矩阵; $\{Q\}$ 为瞬态热激励。对该控制方程可运 用时域积分法进行求解计算 ${ }^{[5]}$ 。

\section{2 红外检测信号快速算法}

有限元控制方程的传统时域积分法求解方法需 要在整个时域上按时间步长 $\Delta t$ 不断迭代求解计算, 为 保证求解的精度, $\Delta t$ 需保持小量, 对于逆问题缺陷重 构中需大量正问题计算时, 其效率不高, 需寻求快速 准确的求解方法。作者研究组提出了针对脱粘缺陷的 红外检测快速计算, 该方法基于频域叠加法和数据库 策略, 将有限元控制方程从时域转化为频域, 并进一 步通过利用无缺陷数据库对方程进行降维处理, 从而 可大大减少求解时间 ${ }^{[6]}$ 。本研究将这一算法拓展到表 面裂纹缺陷的快速数值模拟。

对于有限元控制方程(3), 由于红外响应问题为线 性问题满足叠加原理条件。根据傅里叶变换, 瞬态热 激励 $\{Q\}$ 及温度 $\{T\}$ 可表示为一系列正弦分量的叠加， 因此式(3)可转换为如下形式:

$$
\sum_{n=0}^{N}\left([\boldsymbol{K}]+\mathrm{j} \omega_{n}[\boldsymbol{C}]\right)\left\{\tilde{T}_{n}\right\} \mathrm{e}^{\mathrm{j} \omega_{n} t}=\sum_{n=0}^{N}\left\{\tilde{Q}_{n}\right\} \mathrm{e}^{\mathrm{j} \omega_{n} t}
$$

式中: $N$ 为分解的频率总个数; $\tilde{Q}_{n} 、 \tilde{T}_{n}$ 分别为第 $n$ 个频率所对应正弦分量的幅值; $\omega_{n}$ 为第 $n$ 个谐波角频 率且 $\omega_{n}=n \omega_{\mathrm{b}}$, 其中 $\omega_{\mathrm{b}}$ 是傅里叶分解的基频。有式(4) 可得单个谐波频率分量对应方程为:

$$
\left\{[\boldsymbol{K}]+j \omega_{n}[\boldsymbol{C}]\right\}\left\{\tilde{T}_{n}\right\}=\left\{\tilde{Q}_{n}\right\} \quad n=1,2,3, \cdots N
$$

值得注意的是，对所有谐波分量运用式(5)进行求 解, 会导致很大的计算量。为减少计算量可采取频响 函数插值策略, 即选取合适的频率进行求解, 而对其 他谐波频率则运用插值方法进行近似处理。以上即为 基于频率叠加法的红外快速模拟方法的基本思路。

为进一步提高计算效率, 对于裂纹缺陷导致的红 外信号扰动的快速计算可运用数据库策略。该方法基 本思想是通过提前建立无缺陷模型温度场数据库将 式(5)进行降维处理。对于含裂纹缺陷模型, 可将节点 分为 3 类: 缺陷区域节点、非缺陷区域节点和表面节 点, 只有对缺陷区域节点, 其控制方程(5)中的系数矩 阵 $[K]$ 和 $[C]$ 与无缺陷模型不同, 由此将含缺陷模型的 控制方程与无缺陷模型作差后, 整个控制方程的求解 只与缺陷区域有关, 即可将方程的维数降低至缺陷区 域节点数, 从而大大缩短方程的求解时间。对于裂纹 红外重构问题, 可提前建立无缺陷模型和无缺陷场数 据库, 运用频率叠加法进行求解, 对于重构过程中不 同大小裂纹的正问题计算，只需直接从数据库中取出 相关参数即可, 可进一步提高计算效率。

\section{3 快速算法验证}

为验证上述快速算法的有效性, 建立表面裂纹缺 陷模型并分别运用时域积分法、频域叠加法、数据库 法进行了红外响应信号的计算。在建立模型时, 在距 离裂纹较远的区域所划分网格较大，而在裂纹附近区 域网格划分较密。所用模型的几何形状如图 1 所示, 模型网格参数和材料参数分别列于表 1 与表 2 。

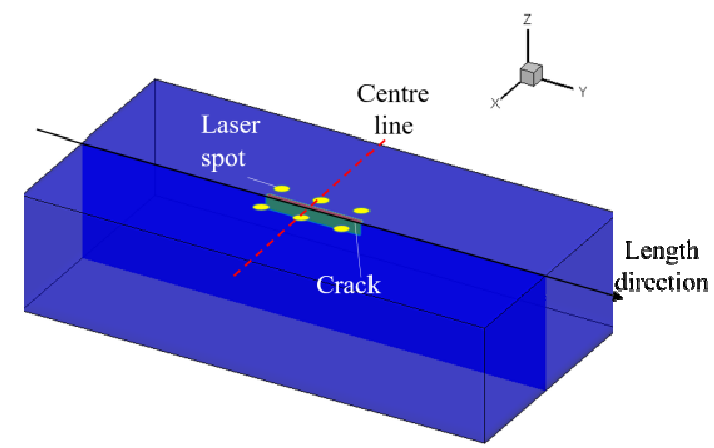

图 1 三维模型图 Fig.1 3D model 
表 1 模型网格参数

Table 1 Parameters of model and mesh

\begin{tabular}{ll}
\hline Parameter & \multicolumn{1}{c}{ Value } \\
\hline Model size & $20 \mathrm{~mm} \times 40 \mathrm{~mm} \times 10 \mathrm{~mm}$ \\
Crack size & $0.2 \mathrm{~mm} \times 8 \mathrm{~mm} \times 1 \mathrm{~mm}$ \\
Mesh size & $0.2 \mathrm{~mm} \times 0.2 \mathrm{~mm} \times 0.1 \mathrm{~mm}$ \\
Element number & 300000 \\
Node number & 320271 \\
\hline
\end{tabular}

表 2 模型材料参数

Table 2 Material parameter of numerical model

\begin{tabular}{lcc}
\hline Region & Crack & Model \\
\hline Material & Air & Aluminum \\
Density $/\left(\mathrm{kg} \cdot \mathrm{m}^{-3}\right)$ & 1.205 & 2769 \\
Specific heat $/\left(\mathrm{J} \cdot \mathrm{kg} \cdot \mathrm{K}^{-1}\right)$ & 1005 & 782.3 \\
Thermal conductivity & & \\
$/\left(\mathrm{W} \cdot \mathrm{m}^{-1} \cdot \mathrm{K}^{-1}\right)$ & 0.025 & 249.5
\end{tabular}

采用的热激励为 $2 \times 3$ 的激光点阵, 即在裂纹两 侧各布置 3 个激励光斑, 光斑直径为 $2.8 \mathrm{~mm}$, 激励函 数为 $g(t)=8 t^{3} \mathrm{e}^{-2 t^{2}}$, 在该激励下试件表面温度在 $1 \mathrm{~s}$ 时刻达到最大值。为保证精度, 时域积分法所采用的 时间步长为 $0.01 \mathrm{~s}$ 。而为提高计算效率, 对频域叠加 法进行了插值处理。在图 2 中给出了不同方法对中心 点温度随时间的变化曲线以及 $1 \mathrm{~s}$ 时中心线上各个节 点的温度分布曲线的计算结果。3 条曲线基本重合, 从时间分布和空间分布两方面 3 种方法的结果具有很 好的一致性, 从而验证了快速算法的有效性。表 3 给 出了不同方法的计算时间, 可以看到在保证计算精度 的同时, 频域叠加法与数据库法相比时域积分法计算

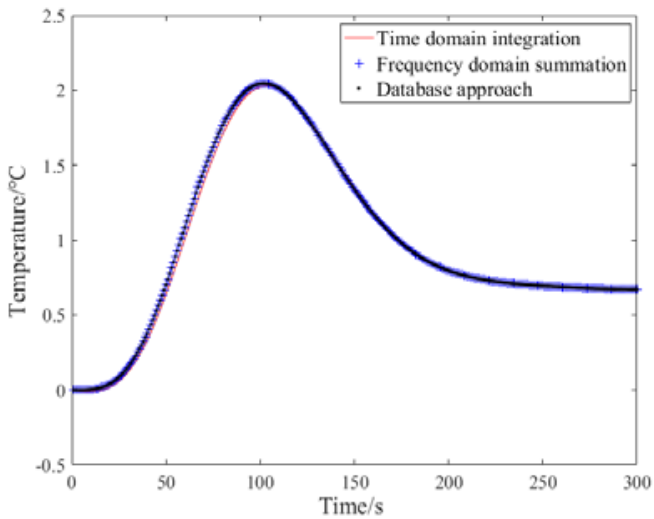

(a) 激励中心温度随时间变化曲线

(a) Transient temperature of excitation center

图 23 种方法计算结果对比

表 33 种方法计算时间对比

\begin{tabular}{cc}
\hline Method & Time domain \\
integration(100 steps)
\end{tabular}

速度大幅提升, 并且数据库法相比频域叠加法具有更 高的计算效率。

\section{2 表面裂纹重构方法}

\section{1 信号特征量选取和重构算法}

影响温度场分布的主要因素有裂纹的长度、宽 度、深度以及裂纹的位置。本文所研究的表面裂纹 宽度很小，一般为 $0.1 \sim 0.4 \mathrm{~mm}$, 裂纹宽度不会对裂 纹两侧区域的温度分布造成显著影响 ${ }^{[7]}$, 而裂纹的位 置一般可利用红外图像分布直接确定, 因此本研究 将裂纹的宽度和位置视为已知, 仅裂纹长度和深度 进行重构。

基于确定论反问题算法, 裂纹重构问题可表述为 计算所得信号特征量与实验所得特征量的加权残差 最小化的优化问题。优化目标函数可表示为如下形 式:

$$
\varepsilon(c)=\sum_{m=1}^{M} w_{m}\left|Z(c)-Z_{m}^{\mathrm{obs}}\right|^{2}
$$

式中: $c$ 为裂纹参数向量; $w_{m}$ 为加权系数; $M$ 为特征 数据点数; $Z_{m}$ obs 为第 $m$ 个特征量数据的实测值。采取 共轭梯度法对裂纹参数进行迭代更新, 当残差达到最 小值时, 即可完成对裂纹的重构。选取合适的特征量 对于重构算法的有效性至关重要。

为此针对宽度 $0.2 \mathrm{~mm}$ 、长度范围 2 6 $\mathrm{mm}$ 、深度 范围 0 2 $\mathrm{mm}$ 的一系列裂纹模型, 对其在第 1 章所示 激光激励下的温度相应进行了计算, 并求取了当表面 温度达到最大值时刻裂纹两侧节点的温差。图 3 为沿

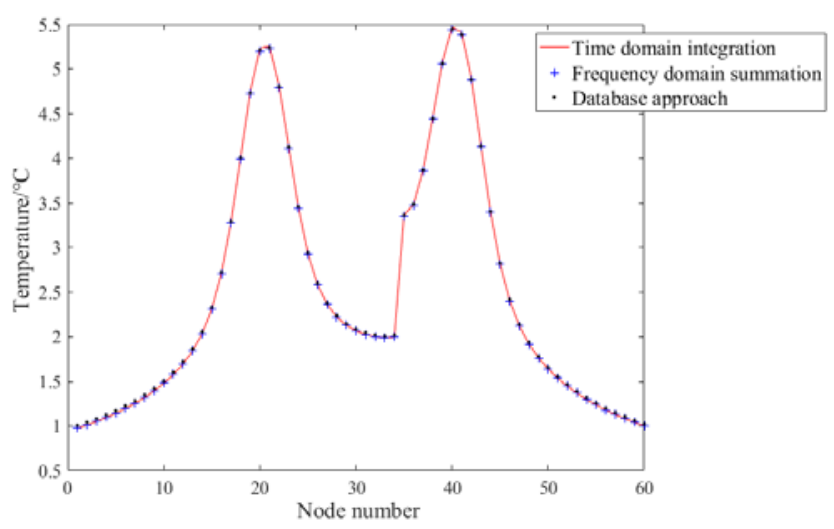

(b) $1 \mathrm{~s}$ 时刻中心线上节点温度变化曲线

(b) Temperature profile of centre line at time instant $1 \mathrm{~s}$

Fig.2 Comparison of calculation results

Table 3 Comparison of time consuming

Frequency domain

summation(interpolation)

Database approach

\begin{tabular}{llll}
\hline Time/s & 2420 & 234 & 119 \\
\hline
\end{tabular}


长度方向裂纹两侧节点温差的分布曲线, 坐标零点对 应裂纹中点位置。对于不同长度的裂纹, 其两侧节点 温差分布有很大差别, 在较短的裂纹长度方向以外区 域, 由于无裂纹存在其两侧节点温差非常小, 而长裂 纹对应的节点温差则远大于该值, 因此各个节点温差 值可以反映长度信息。同时随着裂纹深度的增加, 其 对热流的阻碍也随之增大, 两侧温差增大, 节点温差 值可同时反映深度信息。因此针对该模型选取 30 个 节点温差组成的向量 $\{\Delta T\}$ 作为特征数据对裂纹进行 重构。这时优化目标函数为:

$$
\varepsilon(c)=\sum_{m=1}^{30}\left|\Delta T_{m}(c)-\Delta T_{m}^{\mathrm{obs}}\right|^{2}
$$

式中: $\Delta T^{\mathrm{obs}}$ 为实验得到的节点温差。

为验证该重构方法的有效性, 对裂纹长度分别为 为 $2 \mathrm{~mm} 、 4 \mathrm{~mm} 、 6 \mathrm{~mm}$, 深度分别为 $1 \mathrm{~mm} 、 1.5 \mathrm{~mm}$ 的 6 个裂纹模型的温度响应进行了数值计算, 并添加 $5 \%$ 随机噪声作为模拟实验信号, 提取出相应的特征量 $\{\Delta T\}$ 进行裂纹重构。表 4 为相应重构结果, 重构出的 长度与深度均与实际值基本吻合。

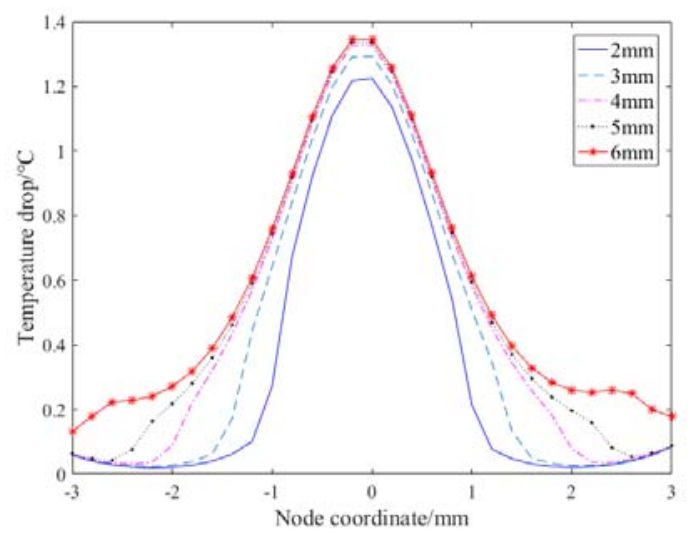

图 3 不同裂纹长度下裂纹两侧温差分布曲线

Fig.3 Temperature difference across crack of different length

\section{2 基于实验信号的裂纹重构}

为进一步验证重构方法的有效性, 进行了激光 红外检测实验, 实验系统如图 4 所示。系统主要由 激光器、产生激励信号的信号发生器和采集红外图 像的红外热像仪组成。裂纹试件如图 5 所示, 裂纹 深度为 $1.5 \mathrm{~mm}$ 、长度 $10 \mathrm{~mm}$ 、宽度 $0.2 \mathrm{~mm}$ 。实验采 用的激励信号为 $2 \mathrm{~s}$ 正弦脉冲信号, 激光器产生单 光斑, 其直径为 $4 \mathrm{~mm}$, 光斑中心距裂纹 $2.8 \mathrm{~mm}$ 。 在 $1.1 \mathrm{~s}$ 时试件表面温度达到最大值, 对此时刻红外
信号进行滤波处理，并按照 2.1 节的重构方法选取 裂纹两侧 24 个节点温差作为特征量进行重构, 节点 间距为 $0.5 \mathrm{~mm}$, 选取的节点区域长度为 $12 \mathrm{~mm}$ 大于 裂纹长度。其重构结果为长度 $8.5 \mathrm{~mm}$, 深度 $1.6 \mathrm{~mm}$, 在深度上可实现较好的重构, 但在长度上重构结果 与实际值出现一定偏差。这是由于不同于模拟时所 采用的点阵激励, 本次实验为单光斑激励, 在裂纹 长度边界附近其两侧温差较小, 在重构中其权重过 小, 需寻找更优的目标函数及特征量来实现长度深 度的准确重构。

表 4 采用计算数据的重构结果

Table 4 Reconstruction results for simulated signals

\begin{tabular}{ccccc}
\hline $\begin{array}{c}\text { Actual value } \\
(\text { length } \times \text { depth }) \\
/ \mathrm{mm}\end{array}$ & $\begin{array}{c}\text { Length } \\
\text { result/mm }\end{array}$ & $\begin{array}{c}\text { Length } \\
\text { error }\end{array}$ & $\begin{array}{c}\text { Depth } \\
\text { result/mm }\end{array}$ & $\begin{array}{c}\text { Depth } \\
\text { error }\end{array}$ \\
\hline $2 \times 1$ & 2.1 & $5.0 \%$ & 1.0 & $0 \%$ \\
$2 \times 1.5$ & 2.1 & $5.0 \%$ & 1.6 & $6.3 \%$ \\
$4 \times 1$ & 4.2 & $5.0 \%$ & 1.1 & $10 \%$ \\
$4 \times 1.5$ & 4.2 & $5.0 \%$ & 1.6 & $6.3 \%$ \\
$6 \times 1$ & 6.3 & $5.0 \%$ & 1.1 & $10 \%$ \\
$6 \times 1.5$ & 6.2 & $3.3 \%$ & 1.4 & $-6.3 \%$ \\
\hline
\end{tabular}

为了寻找合适的特征量, 建立不同裂纹模型, 固 定裂纹深度 $1.5 \mathrm{~mm}$, 分别取裂纹长度 $11 \mathrm{~mm} 、 10 \mathrm{~mm}$ 、 $9 \mathrm{~mm}$ 和 $8 \mathrm{~mm}$ 运用已建立好的数据库进行计算, 研 究其裂纹末端附近的两侧节点温差 (15-24 号测点)。 由图 6 可以看到, 开始时不同长度的裂纹节点温差 几乎一致, 但随着节点编号的增大, 短裂纹的节点 温差开始快速下降并先趋于 0 。虽然在远离激励处, 不同长度裂纹的节点温差都在很小的量级, 但由于 短裂纹的节点温差先接近 0 , 长裂纹的节点温差是 其数倍。对此计算不同长度节点温差与实验值之比 $\max \left\{\Delta T / \Delta T^{\mathrm{obs}}, \Delta T^{\mathrm{obs}} / \Delta T\right\}$, 做出一侧节点温差的比值 图像, 如图 7 所示, 可以看到比值能够较好地反应 出长度的变化。

因此, 为增大裂纹端部温差的权重, 对于两端 测点（1-9 和 16-24 号测点）, 选取计算值与实验值 的比值 $\max \left\{\Delta T / \Delta T^{\mathrm{obs}}, \Delta T^{\mathrm{obs}} / \Delta T\right\}$ 作为特征量, 而中间 测点依旧采用温度差值 $\{\Delta T\}$ 。改良后的优化目标函 数为:

$$
\varepsilon(c)=\sum_{m=1}^{9}\left|\max \left\{\frac{\Delta T_{m}(c)}{\Delta T_{m}^{\mathrm{obs}}}, \frac{\Delta T_{m}^{\mathrm{obs}}}{\Delta T_{m}(c)}\right\}-1\right|^{2}+\sum_{m=10}^{15} 10 \cdot\left|\Delta T_{m}(c)-\Delta T_{m}^{\mathrm{obs}}\right|^{2}+\sum_{m=16}^{24}\left|\max \left\{\frac{\Delta T_{m}(c)}{\Delta T_{m}^{\mathrm{obs}}}, \frac{\Delta T_{m}^{\mathrm{obs}}}{\Delta T_{m}(c)}\right\}-1\right|^{2}
$$




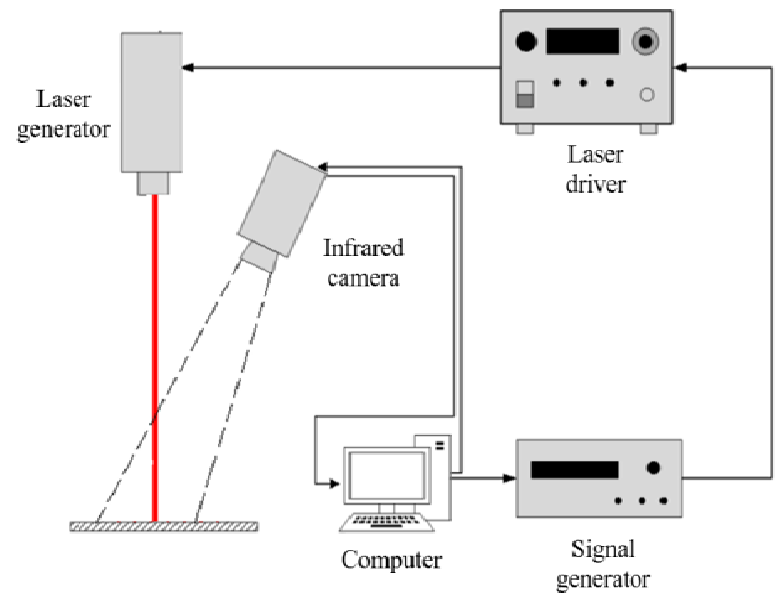

图 4 实验系统示意图

Fig.4 Experimental setup

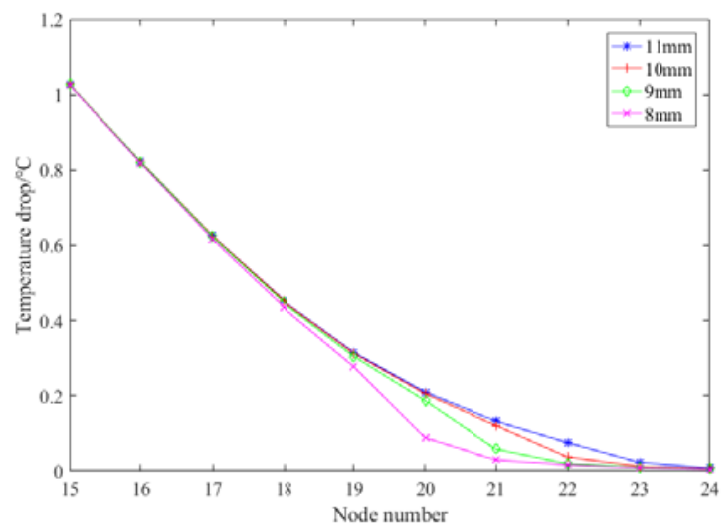

图 6 沿裂纹长度方向的温差分布

Fig.6 Temperature difference distribution along crack

按照式(8)目标函数利用共轭梯度法进行求解, 对 裂纹进行了重构。当设置初始深度 $0.2 \mathrm{~mm}$ 、长度 $6 \mathrm{~mm}$ 时, 所得重构结果为裂纹深度 $1.58 \mathrm{~mm}$ 、长度 $10.6 \mathrm{~mm}$, 与 $1.5 \mathrm{~mm}$ 深、 $10 \mathrm{~mm}$ 长的真实值基本一致。图 8 为 模拟所得的 $1.1 \mathrm{~s}$ 时刻裂纹两侧温差分布曲线, 可以看 到重构裂纹对应检测信号的计算结果与实验结果也 基本一致，进一步验证了重构结果的有效性。

\section{3 结论}

本文基于频率叠加法与数据库策略实现了表面 裂纹激光红外检测信号的快速数值模拟, 并通过与原 有方法的对比验证了快速算法的有效性。针对裂纹长 度与深度的重构, 分别对不同尺寸的裂纹模型表面温 度场进行了计算分析, 选取裂纹两侧节点温差作为重 构特征量, 基于共轭梯度法开发了重构算法并以添加 噪声的模拟温度信号进行裂纹重构, 验证了方法的可

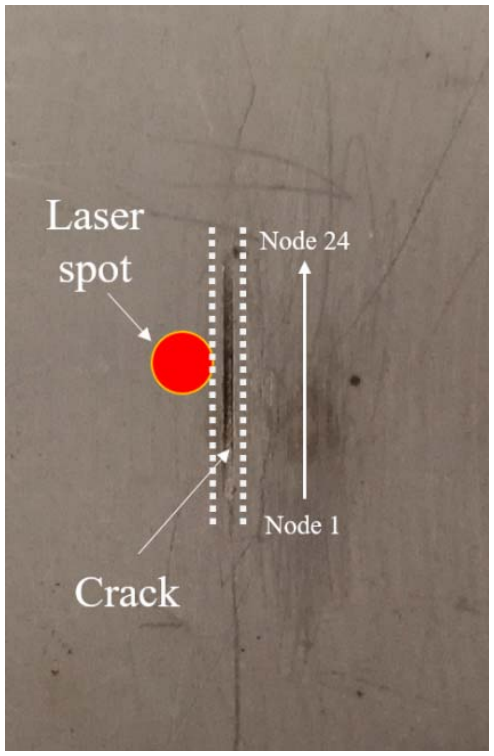

图 5 裂纹试件图

Fig.5 Figure of test-piece with crack

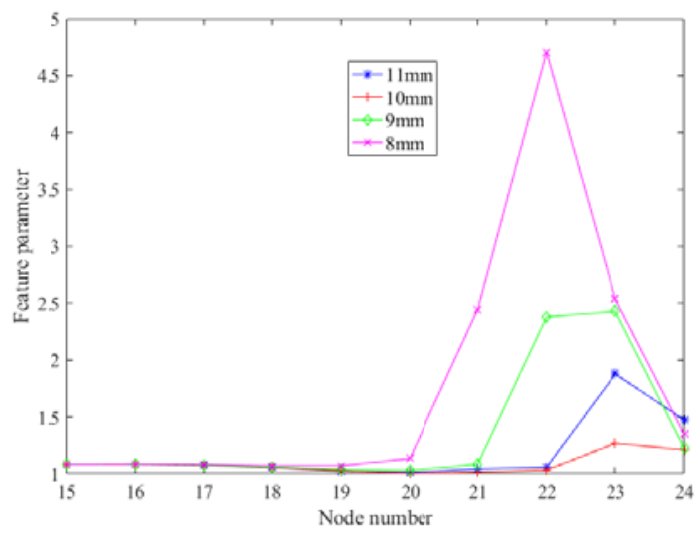

图 7 沿裂纹长度方向的温差特征量分布

Fig.7 Distribution of feature parameter along crack

行性。最后进行了平板试件裂纹红外检测实验, 针对 实验条件的特殊性，对特征量进行更新，实现对基于 实测信号的有效裂纹重构。

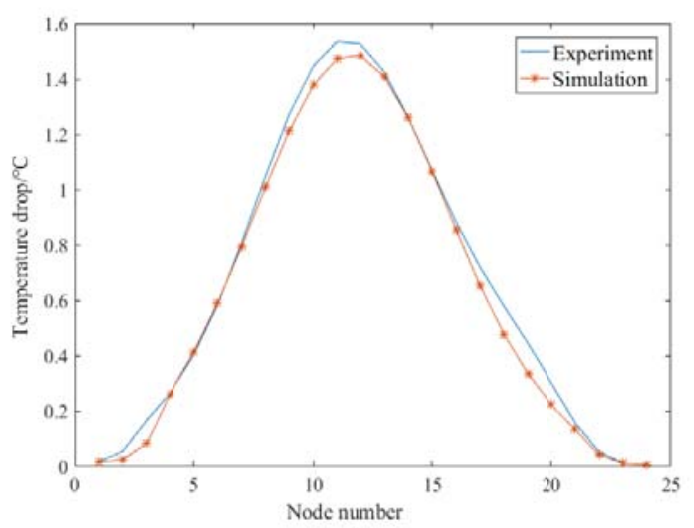

图 $81.1 \mathrm{~s}$ 时刻裂纹两侧温差分布曲线

Fig.8 Temperature difference across crack at time instant $1.1 \mathrm{~s}$ 


\section{参考文献:}

[1] QIU J, PEI C, LIU H, et al. Remote inspection of surface cracks in metallic structures with fiber-guided laser array spots thermography[J]. NDT \& E International, 2017, 92: 213-220.

[2] 戴景民, 汪子君. 红外热成像无损检测技术及其应用现状[J]. 自动化 技术与应用, 2007, 26(1): 10-16.

DAI J, WANG Z. Infrared thermography non-destructive testing technology and its application[J]. Techniques of Automation and Application, 2007, 26(1): 10-16.

[3] AN Y K , Min Kim J , Sohn H. Laser lock-in thermography for detection of surface-breaking fatigue cracks on uncoated steel structures[J]. NDT \& E International, 2014, 65: 54-63.

[4] QIU J, PEI C, LIU H, et al. Quantitative evaluation of surface crack depth with laser spot thermography[J]. International Journal of Fatigue, 2017, 101: $80-85$.

[5] PEI C, QIU J, LIU H, et al. Simulation of surface cracks measurement in first walls by laser spot array thermography[J]. Fusion Engineering and Design, 2016, 109: 1237-1241.

[6] LIU H, XIE S, PEI C, et al. Development of a fast numerical simulator for infrared thermography testing signals of delamination defect in a multilayered plate[J]. IEEE Transactions on Industrial Informatics, 2018, 14(12): 5544-5552.

[7] LI T, Almond D P, Rees D A S. Crack imaging by scanning laser-line thermography and laser-spot thermography[J]. Measurement Science and Technology, 2011, 22(3): 035701.

\section{《红外技术》第八届编辑委员会名单}

荣誉主编: 褚君浩 姜会林 曾 效 曾桂林

主编：苏君红

执行主编：姚立斌

副主编: 刘逸平唐剑李凯朱颖峰陈钱

蔡 毅 何力金伟其 郑云峰 (常务)

编委 (以姓氏笔画为序)

马文坡王双保王霞石峰卢进史衍丽白廷柱白瑜

朱颖峰 刘逸平麦绿波苏君红巫广华李云红李凯李晓峰

杨宇吴一冈吴晗平何力邹继釱汪岳峰张长泉张若岗

张建奇陆卫陈钱陈效双陈福胜金伟其郑云峰郑凯

赵勋杰赵俊胡伟达施加林姚立斌顾国华凌福日 郭杰

唐利斌唐良瑞唐 剑姬荣斌常本康 葛宝臻蒋亚东曾 宇

蔡 毅 熊 平 